\title{
Image-guided endoscopic surgery for spontaneous supratentorial intracerebral hematoma
}

\author{
*Guo-chen Sun, MD, ${ }^{1}$ Xiao-lei Chen, MD, ${ }^{1}$ Yuan-zheng Hou, MD, ${ }^{1}$ Xin-guang Yu, MD, PhD, ${ }^{1}$ \\ Xiao-dong Ma, MD, PhD, ${ }^{1}$ Gang Liu, MD, ${ }^{2}$ Lei Liu, MD, ${ }^{1}$ Jia-shu Zhang, MD, ${ }^{1}$ Hao Tang, MD, ${ }^{1}$ \\ Ru-Yuan Zhu, MD,' Ding-Biao Zhou, MD, PhD, ${ }^{1}$ and Bai-nan Xu, MD, PhD'1 \\ Departments of ${ }^{1}$ Neurosurgery and ${ }^{2}$ Radiology, PLA General Hospital, Beijing, China
}

OBJECTIVE Endoscopic removal of intracerebral hematomas is becoming increasingly common, but there is no standard technique. The authors explored the use of a simple image-guided endoscopic method for removal of spontaneous supratentorial hematomas.

METHODS Virtual reality technology based on a hospital picture archiving and communications systems (PACS) was used in 3D hematoma visualization and surgical planning. Augmented reality based on an Android smartphone app, Sina neurosurgical assist, allowed a projection of the hematoma to be seen on the patient's scalp to facilitate selection of the best trajectory to the center of the hematoma. A obturator and transparent sheath were used to establish a working channel, and an endoscope and a metal suction apparatus were used to remove the hematoma.

RESULTS A total of 25 patients were included in the study, including 18 with putamen hemorrhages and 7 with lobar cerebral hemorrhages. Virtual reality combined with augmented reality helped in achieving the desired position with the obturator and sheath. The median time from the initial surgical incision to completion of closure was 50 minutes (range 40-70 minutes). The actual endoscopic operating time was 30 (range 15-50) minutes. The median blood loss was 80 (range 40-150) ml. No patient experienced postoperative rebleeding. The average hematoma evacuation rate was $97 \%$. The mean ( \pm SD) preoperative Glasgow Coma Scale (GCS) score was $6.7 \pm 3.2$; 1 week after hematoma evacuation the mean GCS score had improved to $11.9 \pm 3.1(p<0.01)$.

CONCLUSIONS Virtual reality using hospital PACS and augmented reality with a smartphone app helped precisely localize hematomas and plan the appropriate endoscopic approach. A transparent sheath helped establish a surgical channel, and an endoscope enabled observation of the hematoma's location to achieve satisfactory hematoma removal.

https://thejns.org/doi/abs/10.3171/2016.7.JNS16932

KEY WORDS endoscopy; hematoma; evacuation; diagnostic and operative techniques

$\mathrm{P}$ ATIENTS with spontaneous supratentorial intracerebral hemorrhage, especially basal ganglia hemorrhage, who require surgery generally have a poor prognosis. Although no apparent benefit from surgery has been reported for these patients, ${ }^{6}$ most patients with this condition actually do undergo surgery. There is currently no single standard surgical treatment, but it has been reported that, in clinical practice, endoscopic hematoma removal has gradually become accepted., ${ }^{7,10,13,17}$ Various surgical methods and tools have been adopted, with differing results. We report here a simple, fast, and economical positioning and surgical procedure for endoscopic evacuation of intracerebral hematomas. The method is based on virtual reality using a hospital's picture archiving and communication system (PACS) and augmented reality using the a smartphone and the Sina neurosurgical assist Android smartphone app, which assists surgeons in precisely placing the obturator and introducing the sheath to the desired target.

\section{Methods \\ Patient Population}

Twenty-five patients with a mean $( \pm$ SD) age of $65.4 \pm$ 11.1 years were included in the study. Of the 25 patients, 18 had experienced a putamen hemorrhage and 7 had had a lobar cerebral hemorrhage. Hematoma volume and residual hematoma volume were calculated with open-access Slicer software. The hematoma evacuation rate, rate of rebleeding, preoperative and 1-week postoperative Glasgow Coma

ABBREVIATIONS GCS = Glasgow Coma Scale; PACS = picture archiving and communication system . SUBMITTED April 13, 2016. ACCEPTED July 8, 2016.

* Drs. Sun and Chen contributed equally to this work. 
Scale (GCS) scores, operative time, endoscopic evacuation time, and blood loss were recorded and analyzed. The study was approved by the PLA General Hospital ethics committee, and informed consent was obtained from all patients' family members.

\section{Inclusion and Exclusion Criteria}

Patients with supratentorial spontaneous intracerebral hemorrhage requiring surgery were eligible for inclusion in this study. Patients with hematoma-induced brainstem herniation were not eligible for the study, because decompressive craniectomy and hematoma evacuation is known to produce superior results. Patients were also excluded if they had a hemorrhage in the brainstem and/or cerebellum, bleeding related to vascular malformation, blood clotting disorders, and/or other contraindications to surgery.

\section{Virtual Reality Image Acquisition}

After admission to the emergency room, all patients underwent $\mathrm{CT}$ to confirm the presence of a cerebral hemorrhage requiring evacuation. Based on the hospital PACS (Philips Healthcare Informatics, Inc.), the hematoma and an outline of the patient's head were reconstructed. Then, an optimal trajectory was identified.

\section{Augmented Reality Image Development}

A reconstructed virtual reality image of the hematoma and the patient's head was imported into the "Sina neurosurgical assist" Android smartphone app (English version downloadable at no cost from the Google Play Store). The virtual reality and augmented reality images were overlapped using surface markers on the patient's head and the smartphone camera. Details of this procedure have been reported in the literature. ${ }^{4,5}$ The optimal trajectory determined in the previous step was then designed on the patient's real head.

\section{Puncture Trajectory Design}

For putamen hemorrhages, we adopted a transfrontal trajectory parallel to the sagittal plane. Using axial CT, we measured the distance between the center of the hematoma and the midline. The entrance point was just behind the frontal hairline and parallel to the midline; the target was the center of the hematoma. The distance and the angle from the puncture site to the center of hematoma were calculated.

For patients with lobar cerebral hemorrhages, based on the location, an individualized, shorter pathway was chosen that was far away from all the brain's eloquent areas.

\section{Endoscopic Hematoma Evacuation Procedure}

We used our patented obturator and transparent sheath set (patent number 201210066281.1, Victor Medical Instruments Co.) to create the working channel. After the obturator was precisely positioned in the center of the hematoma, the inner needle was removed and suction was applied to the hematoma with the syringe to reduce intracranial pressure, and then the transparent sheath was introduced to the ideal depth. Through the transparent sheath, the endoscope (diameter $37 \mathrm{~mm}$; $0^{\circ}$; length $18 \mathrm{~cm}$; Karl Storz) and a metal suction device (diameter $3 \mathrm{~mm}$; length $26 \mathrm{~cm}$; Shanghai Sanyou Medical Instrument Co.) as used to suction the bleeding site, and then a unipolar electric knife was placed against the metal aspirator tube for coagulation.

\section{Imaging Follow-Up}

Cranial CT was performed the day after surgery, and the hematoma evacuation rate was assessed by author Gang Liu, who was blinded to the patients' treatment and clinical outcome, based on the Slicer software, using the method described in our previous study. ${ }^{14}$

\section{Statistical Analysis}

The paired Wilcoxon test was used to compare the preoperative and postoperative GCS scores and hematoma volume. Statistical significance was set at $p<0.05$. All analyses were completed using SPSS statistical software (version 19.0, IBM Corp.).

\section{Results}

There were 25 patients enrolled in the study, including 18 cases of putamen hemorrhage and 7 cases of lobar cerebral hemorrhage. Virtual reality was used in combination with augmented reality to ensure that the optimal trajectory was made and the transparent sheath reached the desired position. Satisfactory removal of the hematoma was achieved in all cases, although the removal was not always complete. In some cases, the decision was made to leave intraventricular hematoma that was hard to see or partial hematoma adjacent to an eloquent area. For patients taking aspirin, patients in whom complete hematoma removal was not achieved, and for patients in whom an intraventricular hematoma remained, a soft drainage tube was placed before wound closure, due to the risk of rebleeding or for residual hematoma drainage.

The median time from the first incision to suture completion was 50 minutes (range 40-70 minutes), and the actual endoscopic operating time was 30 minutes (range 15-50 minutes). Data processing and puncture trajectory design was completed within approximately 5 minutes.

The median blood loss was $80 \mathrm{ml}$ (range $40-150 \mathrm{ml}$ ). No patient experienced postoperative rebleeding. The mean preoperative hematoma volume was $63.9 \pm 26.6 \mathrm{ml}$, and the mean residual hematoma volume was $1.4 \pm 1.8 \mathrm{ml}$, $(\mathrm{p}<0.01)$, representing an average evacuation rate of $97 \%$. The mean preoperative GCS score was $6.7 \pm 3.2$, and the mean GCS score 1 week after hematoma evacuation was $11.9 \pm 3.1$ (statistically significant improvement, $\mathrm{p}<0.01$ ) .

A summary of the patients' demographic and clinical characteristics is provided in Table 1.

\section{Illustrative Cases}

\section{Case 21: A Putamen Hemorrhage}

This 75-year-old man with a history of hypertension was admitted to our hospital after the sudden onset of unconsciousness and paralysis. Emergency cranial CT revealed a right putamen hemorrhage. The distance between 
TABLE 1. Demographic and clinical characteristics of the 25 patients in the study

\begin{tabular}{|c|c|c|c|c|c|c|c|c|}
\hline \multirow[b]{2}{*}{ Case No. } & \multirow[b]{2}{*}{ Age (yrs), Sex } & \multirow[b]{2}{*}{ Hematoma Location } & \multirow[b]{2}{*}{ Side } & \multirow[b]{2}{*}{ Vol (ml) } & \multirow[b]{2}{*}{$\mathrm{RV}(\mathrm{ml})$} & \multirow[b]{2}{*}{$\mathrm{HC}$ to $\mathrm{MD}(\mathrm{mm})$} & \multicolumn{2}{|c|}{ GCS Score } \\
\hline & & & & & & & Preop & 1 Wk Postop \\
\hline 1 & $71, \mathrm{~F}$ & Putamen & $\mathrm{Lt}$ & 121.8 & 5.2 & 34.4 & 3 & 8 \\
\hline 2 & $62, \mathrm{M}$ & Putamen & $\mathrm{Rt}$ & 58.8 & 0 & 22 & 7 & 13 \\
\hline 3 & $64, \mathrm{M}$ & Temporoparietal & Lt & 59.4 & 0 & 35.8 & 10 & 15 \\
\hline 4 & $83, \mathrm{M}$ & Putamen & $\mathrm{Lt}$ & 49.4 & 2 & 30.6 & 11 & 12 \\
\hline 5 & $75, \mathrm{M}$ & Putamen & $\mathrm{Rt}$ & 94.7 & 4.8 & 39.7 & 4 & 8 \\
\hline 6 & $66, \mathrm{M}$ & Putamen & $\mathrm{Rt}$ & 70.8 & 0 & 30 & 4 & 8 \\
\hline 7 & $80, \mathrm{~F}$ & Putamen & $\mathrm{Lt}$ & 41.1 & 3.1 & 30.9 & 4 & 8 \\
\hline 8 & $56, \mathrm{~F}$ & Putamen & $\mathrm{Lt}$ & 37.5 & 5.8 & 33.8 & 12 & 14 \\
\hline 9 & $60, M$ & Putamen & $\mathrm{Rt}$ & 94.8 & 0 & 38.4 & 4 & 15 \\
\hline 10 & $58, \mathrm{M}$ & Putamen & $\mathrm{Rt}$ & 42.7 & 3.3 & 30.8 & 4 & 15 \\
\hline 11 & $52, \mathrm{~F}$ & Putamen & $\mathrm{Rt}$ & 55.2 & 0 & 30.9 & 4 & 9 \\
\hline 12 & $37, \mathrm{M}$ & Putamen & $\mathrm{Rt}$ & 69.2 & 0 & 26.4 & 9 & 15 \\
\hline 13 & $44, \mathrm{M}$ & Putamen & $\mathrm{Rt}$ & 43.1 & 2.1 & 24.9 & 7 & 15 \\
\hline 14 & $73, \mathrm{M}$ & Temporoparietal & Lt & 40.3 & 1.1 & 28.2 & 4 & 9 \\
\hline 15 & $54, \mathrm{M}$ & Frontal & $\mathrm{Lt}$ & 44.2 & 1.5 & 40.1 & 4 & 15 \\
\hline 16 & $70, \mathrm{M}$ & Putamen & $\mathrm{Rt}$ & 82.6 & 0 & 25.3 & 6 & 14 \\
\hline 17 & $64, \mathrm{M}$ & Putamen & $\mathrm{Rt}$ & 42.7 & 1.2 & 36.3 & 6 & 15 \\
\hline 18 & $74, \mathrm{~F}$ & Putamen & $\mathrm{Lt}$ & 42.5 & 0 & 27.8 & 12 & 13 \\
\hline 19 & $67, \mathrm{M}$ & Putamen & $\mathrm{Rt}$ & 69.5 & 0 & 24.9 & 5 & 10 \\
\hline 20 & $64, \mathrm{~F}$ & Putamen & Rt & 39.6 & 0 & 32.3 & 6 & 9 \\
\hline 21 & $75, \mathrm{M}$ & Putamen & $\mathrm{Rt}$ & 140.3 & 1.3 & 26.9 & 4 & 9 \\
\hline 22 & $79, \mathrm{~F}$ & Frontal & $\mathrm{Lt}$ & 46.8 & 2.8 & 18.6 & 12 & 14 \\
\hline 23 & $64, \mathrm{~F}$ & Temporoparietal & $\mathrm{Rt}$ & 63.6 & 0 & 42.8 & 11 & 15 \\
\hline 24 & $61, \mathrm{~F}$ & Temporoparietal & $\mathrm{Rt}$ & 68.2 & 0 & 36.2 & 11 & 14 \\
\hline 25 & $81, \mathrm{~F}$ & Temporoparietal & Rt & 78.7 & 1.8 & 30.5 & 4 & 6 \\
\hline
\end{tabular}

$\mathrm{HC}$ to $\mathrm{MD}=$ hematoma center to midline distance; $\mathrm{RV}=$ residual volume.

the center of the hematoma and the midline was measured and found to be approximately $2.7 \mathrm{~cm}$ (Fig. 1A). Using the hospital's PACS, we achieved a digital 3D reconstruction of the patient's head and right lateral projection of the hematoma (Fig. 1C). Using the Sina smartphone app, we overlapped the resulting combined image and the patient's actual head using the ear, nose, and eye as landmarks. The hematoma's projection on the right side of the patient's head was localized and drawn on the scalp (Fig. 1D). Thus, we designed a transfrontal trajectory parallel to the sagittal plane $2.7 \mathrm{~cm}$ off midline. The entrance point was just behind the frontal hairline, and the target was the hematoma's center; the depth was also measured (7.5 $\mathrm{cm}$ ). A short, straight incision of approximately $4 \mathrm{~cm}$ (Fig. 2A) and a bone flap $2 \mathrm{~cm}$ in diameter (Fig. 2B) were designed. After the dura mater was opened, the local cortex was coagulated, a obturator was inserted into the target, the needle was withdrawn, and the syringe was used to relieve the high cranial pressure from the hematoma; then the sheath was introduced to the hematoma's center. Endoscopic removal of the hematoma was performed with a suction device (Fig. 2C) until all of the hematoma in the visual field was evacuated (Fig. 2D). Cranial CT performed the next day revealed nearly total evacuation of the hematoma (Fig. 1B).

\section{Case 3: A Temporoparietal Cerebral Hemorrhage}

This 64-year-old man was admitted for sudden disturbance of consciousness. Cranial CT demonstrated a temporoparietal cerebral hemorrhage (Fig. 3A and B). Based on the hospital's PACS and the Sina app, we achieved hematoma projection on the left lateral side of the patient's head (Fig. 4A and B). The ideal bur-hole location point was determined by extending a line along the long axis of the hematoma far away from the visual pathway and language-related structures (Fig. 4C). Finally, a trajectory was designed from the superior occipital lobe parallel to the sagittal plane, and the depth and lateral-to-midline distance were measured (Fig. 4D). The rest of the procedure was similar to that described in Case 21. Cranial CT performed the next day revealed total evacuation of the hematoma (Fig. 3C and D).

\section{Discussion}

Whether patients benefit from the evacuation of spontaneous intracerebral hematomas remains unclear, ${ }^{9}$ but removing the hematoma can decrease intracranial pressure and reduce secondary injuries caused by mass effect. Currently, the primary surgical methods for hematoma evacuation are craniotomy and puncture drainage. How- 

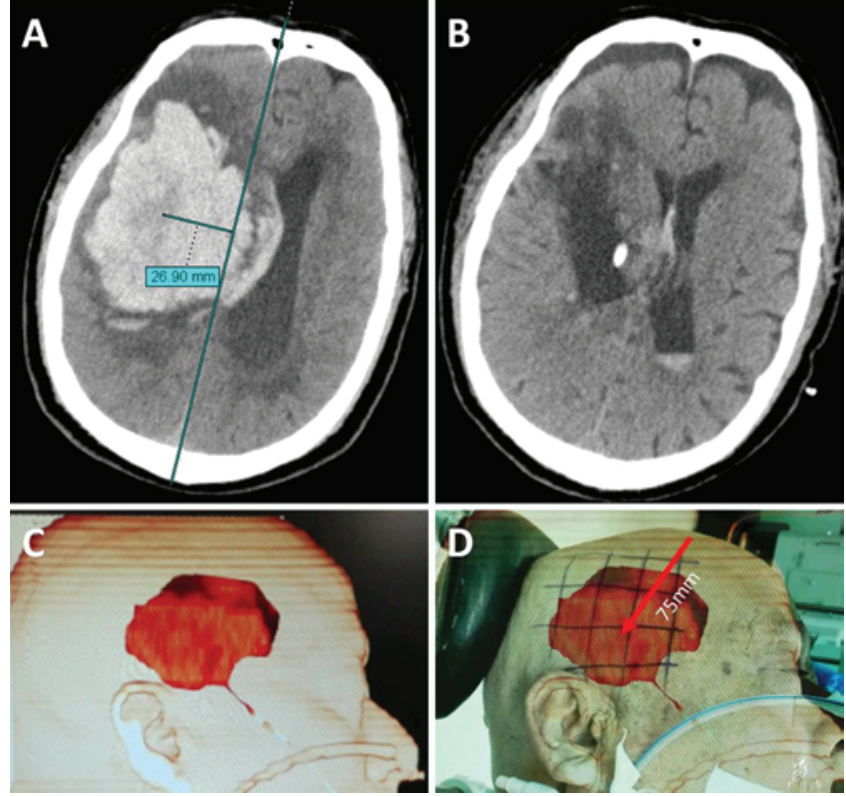

FIG. 1. Case 21. Putamen hematoma case and surgical design. Emergency cranial CT $(\mathbf{A})$ revealed a right basal ganglia hemorrhage with a center-to-midline distance of $26.90 \mathrm{~mm}$. An image of the hematoma in a right lateral view was projected onto a reconstruction of the patient's head (C). Using the Sina neurosurgical assist Android smartphone app, we adjusted the reconstructed head until it matched the patient's actual head in the sagittal view well. The hematoma's projection onto the right side of the patient's head was localized and drawn onto the scalp (D). A puncture trajectory $26.90 \mathrm{~mm}$ in length and parallel to the sagittal plane was designed. The entrance point was just behind the frontal hairline. The target was the center of the hematoma, at a measured depth of 7.5 $\mathrm{cm}$. Postoperative CT revealed that the hematoma was totally evacuated (B). Figure is available in color online only.

ever, it appears that increasing numbers of hospitals are using minimally invasive endoscopic hematoma removal.,11 Some studies have demonstrated that the method is associated with a reduced rate of iatrogenic injury. ${ }^{2}$

Hematoma localization has been based on the surgeon's experience in most reported studies of endoscopic hematoma evacuation, resulting in poor stability, unreliability, and difficulties with quality control. ${ }^{8,12}$ In some studies the hematoma has been localized based on neuronavigation, ${ }^{3,16}$ which can allow the desired position to be accurately reached, but the neuronavigation demands the thinnest layer of CT data. In addition, preparing for navigation is time-consuming, which is a drawback under emergency circumstances.

For endoscopic removal of the hematoma, introducing the sheath to the target precisely and accurately is the key to successful evacuation. In our study, with the help of the simple, fast, and economical procedure described in this article, neurosurgeons obtained satisfactory hematoma positioning and were able to introduce the sheath to the pre-established target; thus, the sheath swing angle was minimized and injury-related adverse effects were further reduced.

Depending on the site of the hematoma, we designed different puncture trajectories to achieve minimal injury to the normal brain: for most putamen hemorrhages, we
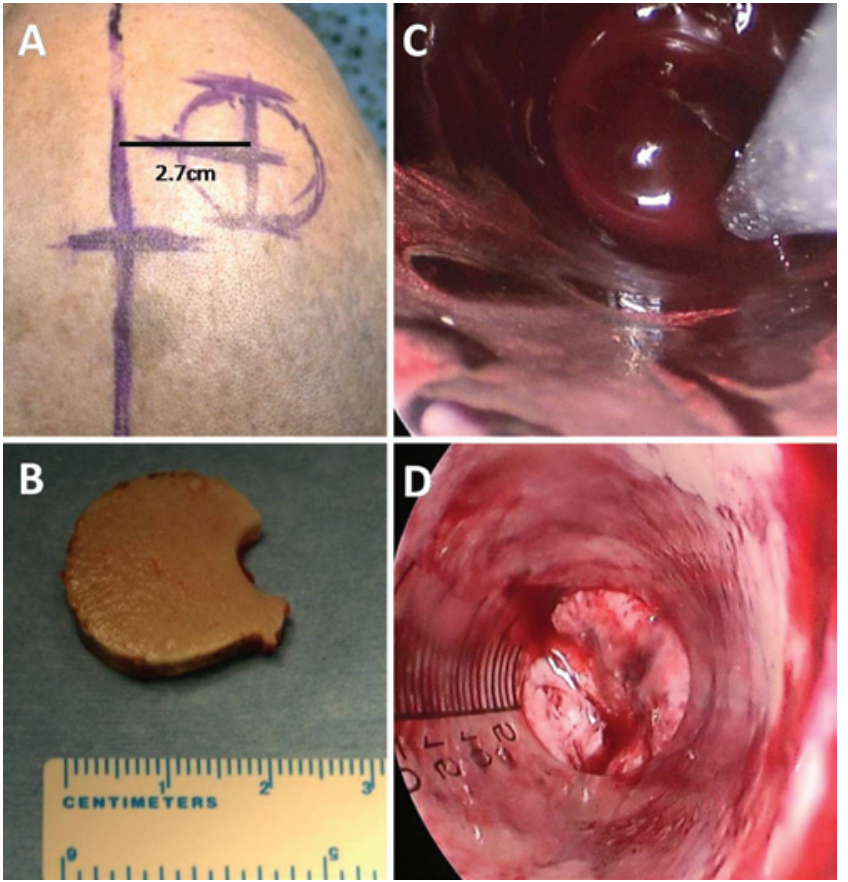

FIG. 2. Case 21. Surgical process of putamen hematoma evacuation. A 4-cm straight incision parallel to the midline $(26.9 \mathrm{~mm})(\mathbf{A})$ with a 2-cm diameter bone flap (B) was designed. Endoscopic removal of the hematoma was performed with a suction device, with observation of the residual hematoma through the transparent sheath $(\mathbf{C})$, until all of the hematoma in the visual field was evacuated (D). Figure is available in color online only.

used a transfrontal trajectory parallel to the sagittal plane. For thalamic hemorrhages, we used a superior occipital puncture trajectory. For lobar hemorrhages, based on the location, a shorter pathway was chosen, tailored to the individual case and taking care to avoid eloquent areas.

Methods of determining the working channel setup have varied in different studies; thus, surgical results have also varied..$^{15}$ In our study, we used our own patented set of instruments. The set consists of a needle (which matches standard syringes), an obturator (diameter $1 \mathrm{~cm}$ ), and a sheath (diameter $1.2 \mathrm{~cm}$ ). According to the depth of the hematoma, sheaths of different lengths can be chosen. This set of instruments is shown in Fig. 5 (upper).

The sheath used in other reports was commonly a cylinder for the entire length, while our sheath had a leading edge that was slightly tapered and had an inclined plane tip. With this shape, the hematoma can easily move into the visual field of the working channel with the help of intracranial pressure.

After the obturator was inserted into the target, we withdrew the needle and used the syringe to evacuate some of the hematoma in order to relieve the high intracranial pressure and confirm placement of the obturator tip in the hematoma, and then the sheath was introduced into the hematoma's center. The transparent sheath provides a working channel for endoscopic and surgical instruments, minimizing iatrogenic injury.

For hematoma evacuation, suction is first applied to the center of the hematoma. The brain tissue surrounding the hematoma and the higher pressure will automatically 

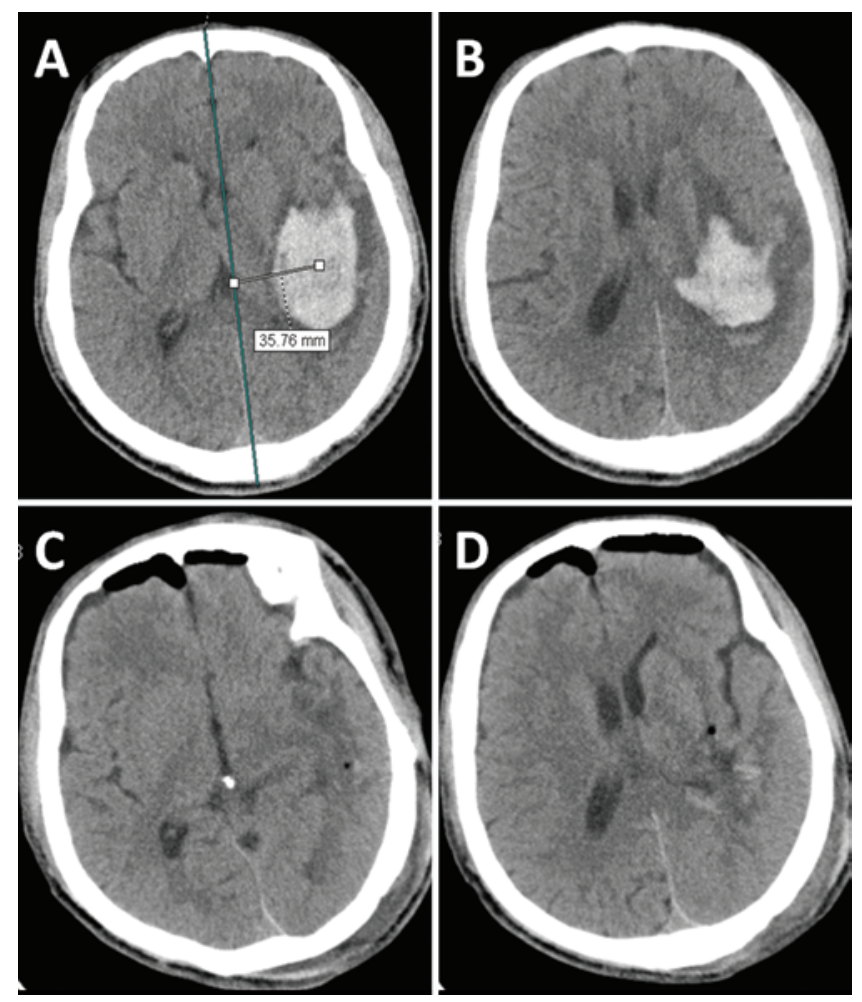

FIG. 3. Case 3. Lobar hematoma and computed tomographic measurement of the hematoma. Preoperative CT demonstrated a temporalparietal cerebral hemorrhage on different axial planes. The hematoma center-to-midline distance is $35.76 \mathrm{~mm}$ ( $\mathbf{A}$ and $\mathbf{B}$ ). A postoperative CT scan performed the next day revealed that the hematoma was totally evacuated ( $\mathbf{C}$ and $\mathbf{D}$ ). Figure is available in color online only.

push the hematoma to the bottom of the working channel, where it can be suctioned easily. Through the transparent side wall of the sheath, the surgeon can easily observe the direction and amount of residual hematoma, which can be removed by slightly swinging the sheath. The process of the evacuation should be from the deep to the shallow portion of the hematoma, and the sheath should be gradually pulled out until the hematoma is removed satisfactorily.

The surgeon should carefully evacuate the hematoma with the suction device to avoid causing damage to brain tissue around the hematoma. If arterial bleeding is observed, the suction device can be moved against the bleeding arterial vessel, and the assistant can use monopolar coagulation to stop the bleeding (see Fig. 5 lower). After satisfactory evacuation of the hematoma, its wall is overlaid by hemostatic gauze. Finally, if no bleeding is observed, the wound is closed.

The time required for craniotomy in this study was approximately 5-10 minutes, the hematoma evacuation time was 15-50 minutes, and the wound closing time was about 10 minutes, so the total operation time was within 70 minutes. As the surgical wound was small, there was less iatrogenic injury, and the amount of intraoperative blood loss was less than $150 \mathrm{ml}$ in all cases. An endoscopically guided sheath swing facilitates the wide observation of the surgical field and reduces the dead area of the view field. In this study, satisfactory hematoma evacuation was achieved in all cases, although in some instances intraventricular
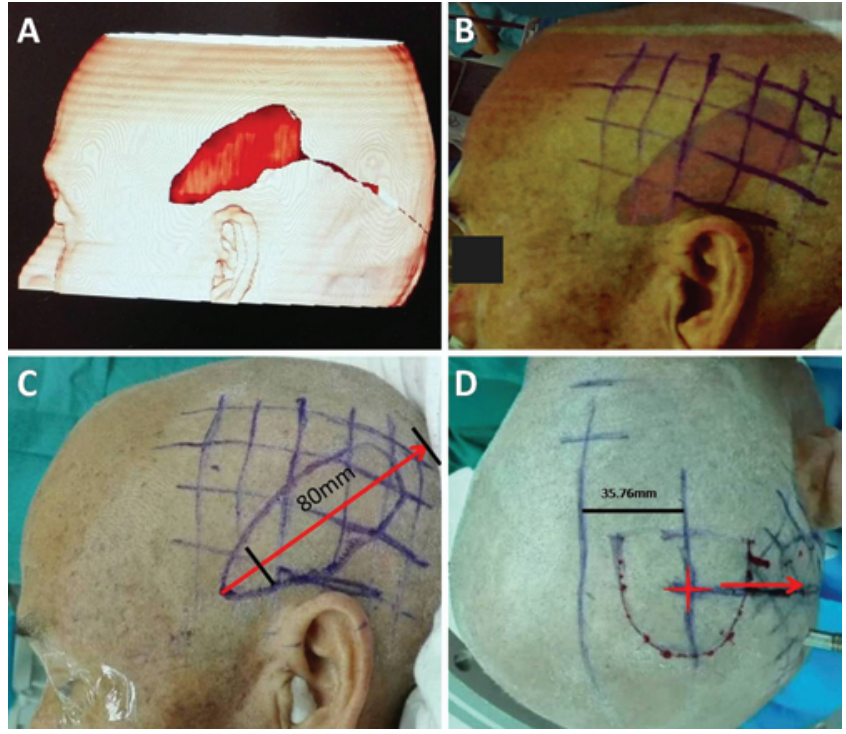

FIG. 4. Case 3. Use of virtual reality and augmented reality guidance for surgical planning. Projection of the hematoma onto the left lateral side of the reconstructed head is achieved (A). After matching of the reconstructed image and the real-time image of the patient's head in the sagittal view using the cell phone camera, the lateral projection of the hematoma on the head is depicted (B). A line (red arrow in $\mathbf{C}$ ) extending along the long axis of the hematoma, far away from the imagined visual pathway and language-related structures, identifies the ideal puncture point. The depth from the entrance point to the target is $80 \mathrm{~mm}(C)$. A puncture trajectory (red arrow in D, lateral-to-midline distance 35.76 $\mathrm{mm}$ ) is designed from the superior occipital region parallel to the sagittal plane. The red cross indicates the entrance point (D). Figure is available in color online only.

hematomas or hematoma adjacent to eloquent areas were not fully removed.

We did not apply commercial neuronavigation (for thinner layer scanning) for hematoma location in this study, and this significantly reduced the time spent in the preparation process. Preoperative surgical planning was easily accomplished. Nearly all hospitals have PACS, smartphones are commonly used worldwide, and the Sina neurosurgical assist is a free Android app with an English version that is downloadable from the Google Play Store. Thus our method reduced barriers to care and saved medical costs.

Although endoscopic surgery for spontaneous supratentorial intracerebral hematoma seems encouraging, the number of patients enrolled in the study was small and there was no control group. A prospective randomized controlled study is needed to provide an evidence-based result.

\section{Conclusions}

The virtual reality and augmented reality techniques used in this study facilitated accurate and precise hematoma positioning, optimal puncture trajectory design, and introduction of a transparent sheath to the center of the hematoma for establishment of a working channel. The use of a transparent sheath for the suction apparatus and endoscope is helpful in identifying hematoma orientation and scope. Its use in combination with virtual reality and augmented reality guidance facilitated hematoma evacuation 


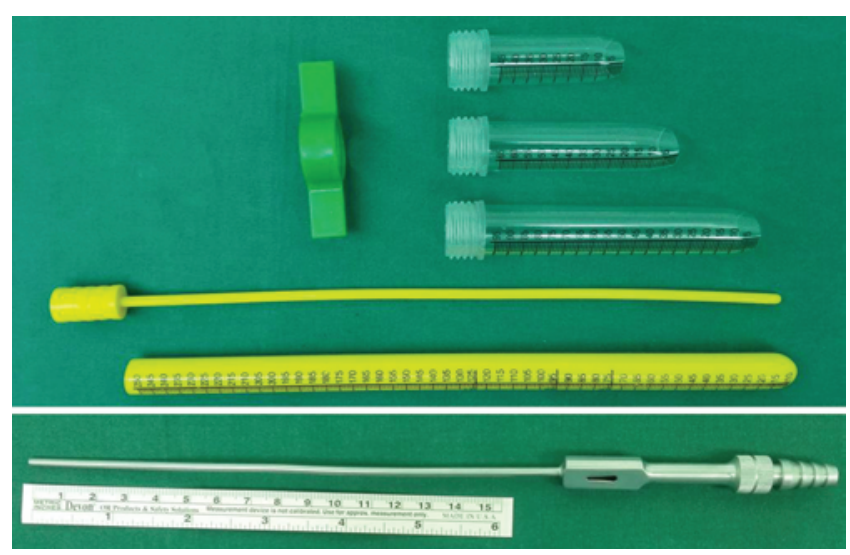

FIG. 5. Tools used for hematoma evacuation. Upper: Surgical tools used in this study consisted of a needle (matched to standard syringes), an obturator (diameter $1 \mathrm{~cm}$ ), and a sheath (outer diameter $1.2 \mathrm{~cm}$ ). According to the depth of the hematoma, various sheaths with different lengths can be used. The end of the sheath has screw threading, which well matches the butterfly handle (green). A snake-like retractor can hold the butterfly handle to stabilize the sheath. Lower: A metal suction apparatus with a diameter of $3 \mathrm{~mm}$ and a length of $26 \mathrm{~cm}$ was used to evacuate the hematoma. For minor intraoperative bleeding, the metal suction device was used to touch the bleeding site, and then a unipolar electric knife was placed against the metal aspirator tube for coagulation. Figure is available in color online only.

via a minimally invasive approach and resulting in good outcomes and relatively short duration of surgery.

\section{Acknowledgments}

We thank the members of the Department of Neurosurgery, PLA General Hospital (Cheng-ye Liu, MD; Hai-hao Gao, MD; Xing-hua Xu, MD; and Yang-rui Zheng, MD), for their collaborative support. This study was funded by grants from the following entities: Science and Technology Projects of Hainan Province (2015SF16), Technological Innovation Foundation of the PLA General Hospital (14KMM37); Medical and Technical Innovation Project, Sanya, Hainan Province (2014YW31); Health Industry Research Project of Hainan Province (14A210218); Natural Science Foundation of Hainan Province (20168362); National Natural Science Foundation of China (81271515); and Key Research and Development Project of Hainan Province (ZDYF2016118).

\section{References}

1. Ausman JI: An important study comparing an endoscopic and a medical approach in treating acute intracerebral hematomas. Surg Neurol 69:437-438, 2008

2. Beynon C, Schiebel P, Bösel J, Unterberg AW, Orakcioglu $\mathrm{B}$ : Minimally invasive endoscopic surgery for treatment of spontaneous intracerebral haematomas. Neurosurg Rev 38:421-428, 2015

3. Ding D, Przybylowski CJ, Starke RM, Sterling Street R, Tyree AE, Webster Crowley R, et al: A minimally invasive anterior skull base approach for evacuation of a basal ganglia hemorrhage. J Clin Neurosci 22:1816-1819, 2015

4. Eftekhar B: App-assisted external ventricular drain insertion. J Neurosurg 125:754-758, 2016

5. Eftekhar B: A smartphone app to assist scalp localization of superficial supratentorial lesions-technical note. World Neurosurg 85:359-363, 2016

6. Fiorella D, Zuckerman SL, Khan IS, Ganesh NK, Mocco J: Intracerebral hemorrhage: A common and devastating dis- ease in need of better treatment. World Neurosurg 84:11361141,2015

7. Hsieh PC: Endoscopic removal of thalamic hematoma: a technical note. Minim Invasive Neurosurg 46:369-371, 2003

8. Hwang SC, Yeo DG, Shin DS, Kim BT: Soft membrane sheath for endoscopic surgery of intracerebral hematomas. World Neurosurg 90:268-272, 2016

9. Mendelow AD, Gregson BA, Rowan EN, Murray GD, Gholkar A, Mitchell PM: Early surgery versus initial conservative treatment in patients with spontaneous supratentorial lobar intracerebral haematomas (STICH II): a randomised trial. Lancet 382:397-408, 2013

10. Nishihara T, Nagata K, Tanaka S, Suzuki Y, Izumi M, Mochizuki Y, et al: Newly developed endoscopic instruments for the removal of intracerebral hematoma. Neurocrit Care 2:67-74, 2005

11. Nishihara T, Teraoka A, Morita A, Ueki K, Takai K, Kirino $\mathrm{T}$ : A transparent sheath for endoscopic surgery and its application in surgical evacuation of spontaneous intracerebral hematomas. Technical note. J Neurosurg 92:1053-1055, 2000

12. Ochalski P, Chivukula S, Shin S, Prevedello D, Engh J: Outcomes after endoscopic port surgery for spontaneous intracerebral hematomas. J Neurol Surg A Cent Eur Neurosurg 75:195-206, 2014

13. Sadahiro H, Nomura S, Goto H, Sugimoto K, Inamura A, Fujiyama Y, et al: Real-time ultrasound-guided endoscopic surgery for putaminal hemorrhage. J Neurosurg 123:1151-1155, 2015

14. Xu X, Chen X, Zhang J, Zheng Y, Sun G, Yu X, et al: Comparison of the Tada formula with software slicer: precise and low-cost method for volume assessment of intracerebral hematoma. Stroke 45:3433-3435, 2014

15. Yamashiro S, Hitoshi Y, Yoshida A, Kuratsu J: Effectiveness of endoscopic surgery for comatose patients with large supratentorial intracerebral hemorrhages. Neurol Med Chir (Tokyo) 55:819-823, 2015

16. Yan YF, Ru DW, Du JR, Shen X, Wang ES, Yao HB: The clinical efficacy of neuronavigation-assisted minimally invasive operation on hypertensive basal ganglia hemorrhage. Eur Rev Med Pharmacol Sci 19:2614-2620, 2015

17. Zhang HZ, Li YP, Yan ZC, Wang XD, She L, Wang XD, et al: Endoscopic evacuation of basal ganglia hemorrhage via keyhole approach using an adjustable cannula in comparison with craniotomy. BioMed Res Int 2014:898762, 2014

\section{Disclosures}

The authors report no conflict of interest concerning the materials or methods used in this study or the findings specified in this paper.

\section{Author Contributions}

Conception and design: Yu, Chen, Ma, Zhou, Xu. Acquisition of data: Yu, Sun, Hou, L Liu, Zhang, Tang, Zhu, Xu. Analysis and interpretation of data: Yu, G Liu, Zhu. Drafting the article: Yu, Sun. Critically revising the article: Yu. Reviewed submitted version of manuscript: Yu. Approved the final version of the manuscript on behalf of all authors: Yu. Statistical analysis: Yu, Sun. Administrative/technical/material support: Yu, Chen. Study supervision: Yu, Chen, Ma, G Liu, Zhou, Xu.

\section{Correspondence}

Xin-guang Yu, Department of Neurosurgery, PLA General Hospital, 28 Fuxing Rd., Beijing 100853, China. email: sjwk8082@126.com. 